Résumés des conférences et travaux

\title{
Histoire et civilisation de l'Écrit en Chine
}

Jean-Pierre Drège

\section{OpenEdition \\ Journals}

\section{Édition électronique}

URL : https://journals.openedition.org/ashp/1218

DOI : $10.4000 /$ ashp. 1218

ISSN : 1969-6310

Éditeur

Publications de l'École Pratique des Hautes Études

\section{Édition imprimée}

Date de publication : 1 octobre 2011

Pagination : 266-267

ISSN : 0766-0677

\section{Référence électronique}

Jean-Pierre Drège, " Histoire et civilisation de l'Écrit en Chine ", Annuaire de l'École pratique des hautes études (EPHE), Section des sciences historiques et philologiques [En ligne], 142 | 2011, mis en ligne le 23 septembre 2011, consulté le 06 juillet 2021. URL : http://journals.openedition.org/ashp/1218 ; DOI : https://doi.org/10.4000/ashp.1218 


\title{
HISTOIRE ET CIVILISATION DE L’ÉCRIT EN CHINE
}

\author{
Directeur d'études : M. Jean-Pierre DrèGE
}

Programme de l'année 2009-2010 : Livres de voyages (suite).

Toutes les conférences de l'année ont été consacrées à l'étude du Yingyai shenglan (Les merveilles des océans) de Ma Huan (vers 1380- après 1451), un musulman originaire de Kuaiji (actuelle Shaoxing au Zhejiang), qui décrit les pays visités par l'amiral Zheng He et sa flotte lors des sept voyages qui l'ont mené jusqu'aux côtes de l'Arabie et de l'Afrique entre 1405 et 1433. Ces expéditions sont bien connues, depuis les travaux de Groeneveldt, de Phillips, de Schlegel, de Rockhill, de Duyvendak et de Pelliot, de Feng Chengjun et de Xiang Da, et plus récemment de Mills et Dreyer. Le Yingyai shenglan forme l'un des trois récits relatifs à ces expéditions, avec le Xiyang fanguo zhi (Relation sur les pays indigènes de l'océan occidental) de Gong Zhen et le Xingcha shenglan (Les sublimes découvertes des nefs sous les étoiles) de Fei Xin. Tous ces ouvrage se suivent de peu : si l'édition du Yingyai shenglan ne date que de 1451, la préface, datée de 1416 semble indiquer qu'une première version du texte existait à cette époque, après que Ma Huan eut participé à un seul des trois voyages qu'il accomplit, entre 1413 et 1415, en tant qu'interprète pour l'arabe et/ou le persan. Il était accompagné de Guo Chongli, lui aussi interprète, et le texte résulte de leurs notes à tous deux. À la suite des autres voyages, en 1421-1422 et 1431-1433, ils procédèrent à des ajouts, notamment sur les Maldives, Dhofar et Aden. Le Xiyang fanguo zhi, quant à lui, a été publié en 1434 et le Xingcha shenglan en 1436.

L'histoire complexe des éditions a été éclaircie, avec son génie habituel, par Pelliot, mais celui-ci n'a pas eu entre les mains toutes les éditions, manuscrites et imprimées, au reste peu nombreuses. Mills, qui publia son Overall Survey of the Ocean's Shore en 1970, prenait pour guide l'édition du Jilu huibian de Shen Jiefu (1533-1601), chapitre 62, suivant en cela le texte établi par Feng Chengjun en 1935. Il divisait les textes en trois groupes, la version du Jilu huibian (augmentée d'un rifacimento), celle du Shengchao yishi de Wu Mikuang datée de 1842 et enfin la copie figurant dans le Guochao diangu de Zhu Dangmian, achevé vers 1542 selon la préface. Tout récemment, en 2005, Wan Ming publia une nouvelle édition annotée, tirée de cette version manuscrite qu'il considère comme la plus proche du texte original (Mingchao ben « Yingyai shenglan » jiaozhu, Les merveilles des océans, un manuscrit des Ming revu et annoté, Pékin, Haiyang chubanshe [Éditions maritimes], 2005). Trois copies manuscrites du Guochao diangu, une collection (congshu) en cent dix chapitres, sont conservées à la Bibliothèque nationale de Chine, dont une seule contient notre texte. Les trois datent des Ming. Celui-ci, retenu par Wan Ming, est mise en concurrence avec trois autres versions manuscrites, celle du Shuoji, un autre congshu des Ming en vingt fascicules, conservé à la bibliothèque de l'Académie des sciences de Chine, celle du 
Dansheng tang de Qi Chenye (1523-1628), qui a servi à l'établissement du texte du Jilu huibian, et enfin celle du Sanbao zhengyi ji, qui fut conservé jadis dans la célèbre bibliothèque du Tianyi ge à Ningbo et qui est maintenant à la Bibliothèque nationale de Chine.

Le texte du Guochao diangu comporte deux préfaces, l'une de Ma Huan, datée de 1416, l'autre signée de Ma Jing et datée de 1444. Celle-ci ne figure pas dans les autres versions. Par contre la postface datée de 1449, qui mentionne la prochaine impression de l'ouvrage, ne figure pas dans l'édition du Guochao diangu.

C'est bien sûr la version du Guochao diangu que nous avons pris pour base de notre cheminement. Toutefois, par prudence, nous avons recherché la copie scannée du texte original à la Bibliothèque nationale et, quoique le texte établi par Wan Ming ne révèle pas d'erreurs bien nombreuses, cette garantie n'a pas été inutile. Au cours de la traduction du texte, on a pris soin de se reporter au Xiyang fanguo zhi (Relation sur les pays indigènes de l'océan occidental) de Gong Zhen dont le texte est bien souvent proche de celui du Yingyai shenglan, mais dans un style plus condensé. Nous n'entrerons pas dans le détail des itinéraires ou des descriptions, qui prendraient ici trop de place et qui seront traités ailleurs, tout au plus signalera-t-on ici que malgré la faible diffusion apparente des livres de voyage, au moins sous une forme imprimée, les voyageurs relativement lettrés avaient une excellente connaissance de leurs prédécesseurs. Ainsi, comme Christophe Colomb se reportant sans cesse à son exemplaire du livre de Marco Polo, Ma Huan, dans sa préface explique qu'il peut confirmer par son livre les choses extraordinaires et étranges décrites par le Daoyi zhilüe (Court mémoire sur les barbares des îles) que laissa Wang Dayuan (1311-?) après ses voyages dans les mêmes océans un peu plus d'un demi-siècle auparavant, un livre que nous avons étudié précisément au cours des années précédentes. 